\section{Theory of RBE}

Abstract

for

Technical Prograss Report

USAEC Contract No. AT(11-1)-1671

1 October 1972

The theory of RBE has been applied to calculate the effect of a mixed radiation enviroment on detectors, dosimeters and cells. This has made it possible to calculate the response of biological cells, for example, to beams of heavy ions, neutrons, and stopped pions. The theory provides the functional form of the response of these detectors to a radiation field once the field is specified and a compact set of radiation parameters, different for different detectors, has been determined from experiment. Using knowledge of the secondary particle spectrum in tissue and cellular parameters determined from earlier work, the calculation. of the survival of kidney, Hela, and leukemia cells after irradiation with $14 \mathrm{MeV}$ neutrons is in good agreement with experiment. At the Princeton Particle Accelerator, an experiment on the survival of mammalian cells was found to be in excellent agreement with predictions of the theory, in the Bragg peak of a $4 \mathrm{MeV}$ nitrogen beam. These results imply that the theory of $\mathrm{RBE}$ is essentially correct in principle 


\section{DISCLAIMER}

This report was prepared as an account of work sponsored by an agency of the United States Government. Neither the United States Government nor any agency Thereof, nor any of their employees, makes any warranty, express or implied, or assumes any legal liability or responsibility for the accuracy, completeness, or usefulness of any information, apparatus, product, or process disclosed, or represents that its use would not infringe privately owned rights. Reference herein to any specific commercial product, process, or service by trade name, trademark, manufacturer, or otherwise does not necessarily constitute or imply its endorsement, recommendation, or favoring by the United States Government or any agency thereof. The views and opinions of authors expressed herein do not necessarily state or reflect those of the United States Government or any agency thereof. 


\section{DISCLAIMER}

Portions of this document may be illegible in electronic image products. Images are produced from the best available original document. 


\section{THEORY OF RBE \\ Technical Progress Report \\ AEC Contract No. AT $(11-1)-1671$ \\ 1 October 1972}

1) There has been no deviation in program from that proposed in the renewal application, either in the technical content or in the level of expenditure. The principal investigator has spent, or will spend,. the following time on the project:

0.5 time. 1 January-31 May (while on research leave at Hammersmith Hospital, London).

1.0 time $\quad 1$ June-3l July

0.3 time 1 september-31 December

2) Following is an index of papers and reports:

Coo-1671-34 R. Katz, S. C. Sharma, and M. Homayoonfar, Detection of Energetic Heavy Ions, Nucl. Instr. and Meth. 100, 13-22 (1972). c00-1671-36 T. E. Furtak and R. Katz, Simulation of Particle Tracks in Emulsion, Radiation Effects 11, 195-199 (1971).

Coo-1671-38 R. Katz, S. C. Sharma, and M. Homayoonfar, Irradiation Equivalence, Health Physics

c00-1671-39 R. Katz, Track Structure Theory in Dosimetry, in Symposium on Dosimetry Techniques Applied to Agriculture, Industry, Biology, and Medicine, IAEA, Vienna

Coo-1671-40 R. Katz and S. C. Sharma, Response of Detectors to a Mixed Radiation Environment, Symposium on Neutronendosimetrie in Biologie und Medizin, Munich (1972). Euratom 
Co0-1671-41 R. Katz, S. C. Sharma, and M. Homayoonfar, LET Effects in 1-Hit Detectors a) Radical Production in Solid Biological Substances, and b) the Fricke Dosimeter. proceedings of the Third Symposium on Microdosimetry, Stresa, Italy, (1971), H. G. Ebert, ed., Eur 4810 d-f-e, Euratom, Luxembourg. pp 199-215. Coo-1671-4.2 R. Katz, S. C. Sharma, and M. Homayoonfar, Cellular Inactivation by Heavy Ions, Neutrons, and Pions, Proceedings of the Third Symposium on Microdosimetry, Stresa, Italy, (1971), H. G. Ebert, ed., Eur 4810 d-f-e, Euratom, Luxembourg pp 267-287. Coo-1671-43 R. Katz, S. C. Sharma and M. Homayoonfar, The Structure of Particle Tracks, Chapt. 6 in Topics in Radiation Dosimetry, F. H. Attix, ed., Academic Press, N.Y.

coo-1671-44 This Report

Coo-1671-45 Trienniel Report to the Division of Biology and Medicine 3) The principal impact of the work of the past year has been in the analysis of the effect of a mixed radiation environment on detectors, dosimeters, and cells. This has made it possible to calculate the response of cells to beams of heavy ions, neutrons, and stopped pions, and to make similar calculations dealing with the response of detectors and dosimeters. Methods have been developed for including the present theory of track struture in Monte Carlo calculations dealing with surir traisport problems. The results obtained have implications for dosimetry and radiation protection, as well as for the understanding of fundamental aspects of track structure and detector and cellular response to radiation. 
The theory of detector and cellular response yields the functional form of the response of 1-hit detectors and dosimeters, and a number of cells, from theoretical relations describing the spatial distribution of delta rays produced along particle tracks, and by considering the structure of tracks in emulsions which do not accumulate "sub-lethal". damage, and then by extending the conceptual structure of the theory to detectors which can accumulate sub-lethal damage. The radiosensitivity parameters of both 1 -hit detectors and cells must be determined experimentally, for each type of detector, and compactly represent the response of the detector to radiation. Once the parameters have been determined the theory yields a prediction of the response of the detector to any specified radiation environment.

Calculations of the response of both $\mathrm{T}-1$ kidney cells, and Hela cells to $1.4 \mathrm{MeV}$ neutrons have been made using a calculated secondary particle spectrum due to Caswell and Coyne, with good agreement between calculated and measured survival curves for these cells.

A calculation of the response of the Fricke dosimeter to $14 \mathrm{MeV}$ neutrons has been made, using the secondary particle spectrum in tissue (as an approximation to water), with good results.

In order to extend these calculations to other neutron spectra and other detectors, it is important that the calculations of caswell and Coyne be extended to other neutron energies, and to the materials from wich detectors and dosimeters are made.

Alternatively, the detector model may be. incorporated into Monte Carlo radiation transport codes to examine both cellular and dosimeter response in radiation environments of interest. A calculation for $14 \mathrm{MeV}$ neutrons has been made by T. W. Armstrong, Oak Ridge National Laboratory (ORNL-TM-3816), incorporating the present theory of cellular response. 
A more significant verification of the theory of cellular survival in a mixed radiation environment has been made at the Princeton Particle Accelerator (Prediction of the Spatial Distribution of Cell Survival in Heavy Ion Beams, K. G. Vosburgh, Science 174, 1125-27 (1971) .)

The prescription for cellular survival in a mixed radiation environment and the cellular radiation parameters found in the present investigations [COO $1671-27,-35,-42]$ were incorporated into a Monte-Carlo description of a $3.9 \mathrm{GeV}$ nitrogen beam, to yield calculations of cellular survival in the Bragg peak of the beam, for comparison with measurement of cellular survival made in a phantom. According to Vosburgh, "The experimental verification of this method. for predicting cell survival after heavy ion irradiation lends support to this method for predicting biological responses in particular untested situations."

Though many aspects of the theoretical and experimental pictures need to be clarified and filled in, the theory appears applicable to photographic emulsion; scintillation counters, thermoluminescent dosimeters, radical formation, the Fricke dosimeter, enzymes, viruses, bacterial spores, bacteria, yeast, diseased and normal mammalian cells. It is not applicable to detectors where the observed end-point is produced by nuclear collisions, but is restricted to systems in which electromagnetic interactions predominate. It is an important and fundamental result of the present theory that RBE effects (variation in response with LET of the bombarding particle, or with "radiation quality") are basically physical rather than biological. We have no need to postulate that biological areils9mposediof elements in difient ways to radiations of different quality: The variation of RBE with LET is linked with the spatial aspects of the deposition of energy by delta-rays, and the accumulation (or non-accumulation) of sub-lethal damage by the sensitive elements of the detector. 
A result which may prove important in radiation protection and dosimetry is that insensitive 1 -hit detectors respond to particles of differing charge and energy in a way which is distinctly different from the response of sensitive l-hit detectors. While insensitive detectors yield a response which is essentially single valued in LET, sensitive detectors are multiple valued in LET. Thus two particles having the same LET respond differently, with the particle of higher charge yielding the greater response. Thus combinations of sensitive and insensitive detectors can be employed to identify the charge and velocity of a single fast particle which passes through both detectors, or to disentangle the LET spectrum into its charge components; when used in conjunction with the theory (CO0-1671-39). Using the output of this analysis as the input to the theory of cellular survival in a mixed radiation environment, we anticipate that a dosimeter can be developed whose output will be given as an estimate of the survival of mammalian cells. It seems also possible to produce simpler dosimeters which can give meaningful estimates of "radiation quality", to the extent that such a term is meaningful. A new way to characterize radiation environments is to estimate the "equivalent irradiation", or its "equivalent ion-kill probability" (COO-1671-38), as the monoenergetic track-segment irradiation giving the same survival curve as the observed mixed radiation field. According to the theory, the effect of the irradiation of a cell culture (specific for both the culture and the conditions of irradiation- oxygen, temperature, culture conditions, etc.) is determined. When we know the fraction of the dose which is delivered in the gamma-kill mode. 
The present model of track structure and detector response provides a new set of coordinates against which to test models of the behavior of any particular detector. In a formal way the theory of the response of 1 -hit detectors has shown that two numbers, the $D-37$ dose and the radius, $a_{\circ}$, of a sensitive volume, are capable of predicting the response of these detectors to radiations of different LET. In specific cases we must ask what the numbers mean, and indeed. how does the concept of a D-37 dose apply to a particular system.

In the application of the theory to scintillation counters, a saturation of response with increasing dose of gamma rays was postulated, though it had never been observed, and it was shown that a characteristic radius was demanded by the model. It was suggested that the characteristic saturation would be observed only with pulses of gamma-rays short compared to the lifetime of the excited state responsible for photon emission, and that the characteristic radius required by the theory could be associated with exciton diffusion. Following the direction given by our earlier paper (c00-1671-13), a recent paper (On the Response of Impurity Activated Alkali Halide Scintillators, M. Ladu, M. Pelliccioni, and M. Roccella, Nucl, Instr. and Methods 101, 383-9 (1972) ) has shown that improved agreement between theory and experiment results from the explicit introduction of exciton diffusion as a mechanism for energy transfer.

In the application of the theory to the Fricke dosimeter, the parallel response of the dosimeter to heavy ions to the response of scintillators to heavy ions suggests a similar mechanism. To one extent, the prediction of the theory that the response of the Fricke dosimeter must exhibit a saturation effect at high gamma-ray doses 
(not related to oxygen or ferrous ion depletion), is consistent with observations on the response of the dosimeter to nanosecond pulses made recently in Munich by Dr. L. Schachinger. We therefore anticipate the confirmation of our suggestion that energy transfer in water, possibly by excitons, is responsible for the "sensitive volume" demanded by the formal application of the track structure theory to the decline in yield of the Fricke dosimeter with the increase of the LET of bombarding particles $[$ coo-1671-39,41].

As the model is applied to an ever increasing number of phenomena, we expect an increasing interplay of both conceptual and quantitative results. For example, we expect that numbers determined for the $D-37$ dose in radical production in both dry chemical systems and in aqueous solutions to be able to speak to the question as to the importance of radical production in cellular inactivation, For example, the quantitative demonstration of the agreement of theory and experiment for the track width regime in emulsion has led to the concept of a track width regime in cellular radiobiology. The association of an RBE with sub lethal damage in cells now motivates the search for the identification of cumulative damage in physical detectors, on the part of the present investigation.

It is the clear implication of the present model that the"RBE" of 1-hit detectors must be less than 1 , and that the most sensitive detector must have the lowest RBE. This observation has led to the suggestion that tissue equivalent 1-hit detectors be exposed to measured doses of gamma rays and neutrons at different laboratories as a test of their neutron dosimetry [000-1671-40], as a direct application of the present theory to a pressing dosimetric problem. 